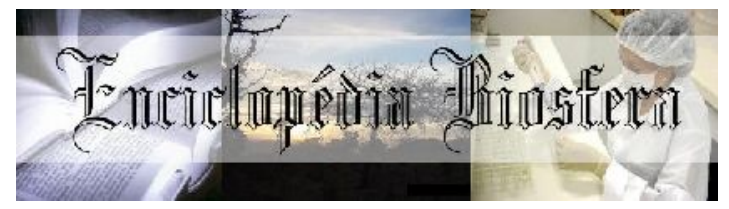

\title{
CONHECIMENTO SOBRE TOXOPLASMOSE ENTRE GESTANTES DE UMA UNIDADE BÁSICA DE SAÚDE DA AMAZÔNIA OCIDENTAL BRASILEIRA
}

\author{
Marcos Henrique Oliveira de Sousa ${ }^{1}$, Reilson Mendes de Almeida ${ }^{1}$, \\ Klismann de Araújo Freire ${ }^{1}$, Eder Ferreira de Arruda ${ }^{2}$, Janete Lemos Andrade ${ }^{2}$ \\ 1. Acadêmico do Curso de Enfermagem e Bolsista do Programa de Iniciação Científica (PIC) \\ do Centro Universitário UNINORTE, Rio Branco - AC, Brasil. \\ 2. Docente do Centro Universitário UNINORTE, Rio Branco - AC, Brasil. \\ E-mail: ederarrud@gmail.com.
}

Recebido em: 04/10/2019 - Aprovado em: 30/11/2019 - Publicado em: 15/12/2019

DOI: 10.18677/EnciBio_2019B12

A toxoplasmose é uma doença infecciosa causada por um protozoário chamado Toxoplasma gondii. Quando a infecção é contraída durante o período gestacional pode ser transmitida ao feto causando graves consequências. Esse trabalho teve como objetivo identificar o conhecimento sobre toxoplasmose entre gestantes atendidas em uma unidade básica de saúde do município de Rio Branco, Acre. Tratou-se de um estudo transversal realizado através da aplicação de um questionário sobre as características sociodemográficas e conhecimentos sobre a toxoplasmose de 70 gestantes nos meses de julho e agosto de 2018. Observou-se que a maioria das grávidas se encontrava na faixa etária de 18 a 24 anos (48,6\%), se declarava de cor parda e tinha o ensino médio $(60,0 \%)$, com companheiro $(71,4 \%)$, não exercia atividade remunerada $(68,6 \%)$ e tinha renda familiar maior que um salário mínimo (42,9\%). Com relação aos conhecimentos sobre a toxoplasmose, a maior parte desconhecia às formas de transmissão $(51,4 \%)$, complicações $(72,9 \%)$, meios de diagnóstico $(57,1 \%)$, profilaxia e tratamento $(74,3 \%)$, medidas preventivas $(52,9 \%)$ e não receberam orientações sobre a doença durante a assistência pré-natal $(61,4 \%)$. Desse modo, os resultados apontam que existe entre as gestantes falta de informação em relação à toxoplasmose, sendo fundamental que os profissionais e os serviços de saúde estabeleçam medidas de orientação sobre os meios de transmissão e controle da doença.

PALAVRAS-CHAVE: Atenção Primária à Saúde; Gestantes; Toxoplasmose.

\section{TOXOPLASMOSIS KNOWLEDGE AMONG PREGNANT WOMEN IN A BASIC BRAZILIAN AMAZON HEALTH UNIT}

\begin{abstract}
Toxoplasmosis is an infectious disease caused by a protozoan called Toxoplasma gondii. When the infection is contracted during gestation it can be transmitted to the fetus causing serious consequences. This study aimed to identify the knowledge about toxoplasmosis among pregnant women attended at a basic health unit in the city of Rio Branco, Acre. This was a cross-sectional study conducted through a questionnaire on the sociodemographic characteristics and toxoplasmosis knowledge of 70 pregnant women in July and August 2018. It was observed that most of the pregnant women were in the age group of 18 years. 24 -year-old (48.6\%), declared
\end{abstract}


himself brown and had high school $(60.0 \%)$, with partner $(71.4 \%)$, had no paid activity $(68.6 \%)$ and had family income greater than 1 minimum wage $(42.9 \%)$. Regarding knowledge about toxoplasmosis, most were unaware of the forms of transmission (51.4\%), complications (72.9\%), means of diagnosis $(57.1 \%)$, prophylaxis and treatment $(74.3 \%)$, preventive measures $(52.9 \%)$ and received no guidance on the disease during prenatal care (61.4\%). Thus, the results indicate that there is a lack of information among pregnant women regarding toxoplasmosis, and it is essential that health professionals and services establish guidance on the means of transmission and control of the disease.

KEYWORDS: Primary Health Care; Pregnant women; Toxoplasmosis.

\section{INTRODUÇÃO}

A toxoplasmose é uma zoonose de distribuição mundial, causada pelo protozoário Toxoplasma gondii. Apresenta quadro clínico variado, desde infecção assintomática a manifestações sistêmicas extremamente graves e pode ocorrer em qualquer fase da vida do indivíduo, inclusive no período da gravidez (BRASIL, 2010).

A infecção durante a gestação ocorre por via oral por meio da ingestão de cistos teciduais presentes em carnes cruas ou mal cozidas e/ou pelo consumo de outros alimentos contaminados com oocistos do parasito dispersos na água ou solo (MONTOYA; REMINGTON, 2008).

A maior parte dos casos de toxoplasmose gestacional não apresenta sintomatologia ou apresenta sintomas comuns a outras doenças, por isso geralmente não é detectada de forma oportuna para o tratamento precoce e adequado (BRASIL, 2018).

Quando a doença não é diagnosticada precocemente, principalmente durante os dois primeiros trimestres da gestação, normalmente, ocorre à transmissão intrauterina, que pode ocasionar aborto espontâneo, nascimento prematuro, morte neonatal, ou sequelas severas no feto (AMENDOEIRA; CAMILLO-COURA, 2010).

Neste contexto, a identificação do conhecimento das gestantes sobre a toxoplasmose permite o planejamento de ações e atividades de educação em saúde adequadas, focando nos pontos críticos, no intuito de prevenir a transmissão da doença e também proporcionar o tratamento oportuno, caso haja infecção na gestação. Dessa forma, o presente estudo teve como objetivo identificar o conhecimento sobre toxoplasmose entre gestantes atendidas em uma unidade básica de saúde do município de Rio Branco, Acre.

\section{MATERIAL E MÉTODOS}

Tratou-se de um estudo observacional do tipo transversal com 70 gestantes atendidas em uma unidade básica no município de Rio Branco-Acre. O município de Rio Branco-Acre (Latitude: 958'26"S; Longitude: 6748'27"O) está localizado na Amazônia Sul Ocidental, possui uma área territorial de $8.834,942$ km², densidade demográfica estimada em $38,03 \mathrm{hab} / \mathrm{km}^{2}$ e uma população estimada de 401.155 pessoas (IBGE, 2017).

Para o cálculo amostral foi considerada a média mensal de grávidas que realizaram consultas pré-natais na referida unidade no ano de 2017 , com intervalo de confiança de 95,0\% e um percentual de margem de erro de 5,0\%, o que determinou uma amostragem mínima de 70 mulheres. As gestantes foram selecionadas, de forma aleatória, entre as mulheres que realizam consultas prénatais na unidade no período de julho e agosto de 2018. 
Foram incluídas, as grávidas que estavam entre o primeiro e segundo trimestre gestacional e com idade igual ou superior a 18 anos. Foram excluídas as que não aceitaram participar da pesquisa e as que não se sentiam em condições físicas e psicológicas para responder as perguntas.

Para a coleta de dados, foi aplicado um questionário semiestruturado sobre as características sociodemográficas e conhecimentos sobre a toxoplasmose. $\mathrm{O}$ programa estatístico Statistical Package for the Social Sciences (SPSS), versão 21.0, foi utilizado para digitação, revisão e análise dos dados. Neste, foram calculadas as frequências absolutas e relativas das variáveis de interesse.

A presente pesquisa foi aprovada pelo Comitê de Ética em Pesquisa da União Educacional do Norte - UNINORTE, com o parecer № 2.634.540 e CAAE № 86979318.2.0000.8028.

\section{RESULTADOS E DISCUSSÃO}

A maioria das gestantes estava na faixa etária de 18 a 24 anos (48,6\%), (Tabela 1). De igual modo, na pesquisa de Moura et al. (2016) sobre o perfil epidemiológico e conhecimento das gestantes atendidas na unidade básica de saúde de Satuba - AL, verificou-se que a maior parte das gestantes tinha menos de 25 anos.

TABELA 1 - Características sociodemográficas de gestantes atendidas em uma unidade de atenção primária a saúde de Rio Branco, Acre, Brasil, 2018.

\begin{tabular}{lcc}
\hline Variável & N & $\%$ \\
\hline Faixa etária (anos) & & \\
18-24 & 34 & 48,6 \\
$25-29$ & 18 & 25,7 \\
$30-34$ & 13 & 18,6 \\
$35-40$ & 4 & 5,7 \\
>40 & 1 & 1,4 \\
Cor/ Raça & & \\
Parda & 42 & 60,0 \\
Negra & 12 & 17,1 \\
Branca & 15 & 21,4 \\
Amarela & 1 & 1,5 \\
Escolaridade & & \\
Não alfabetizada & 1 & 1,4 \\
Ensino Fundamental & 20 & 28,6 \\
Ensino Médio & 42 & 60,0 \\
Ensino Superior & 7 & 10,0 \\
Situação Conjugal & & \\
Com companheiro & 50 & 71,4 \\
Sem companheiro & 20 & 28,6 \\
Atividade remunerada & & \\
Sim & 22 & 31,4 \\
Não & 48 & 68,6 \\
Renda familiar* & & \\
< SM & 18 & 25,7 \\
1 SM & 22 & 31,4 \\
> SM & 30 & 42,9 \\
\hline Total & $\mathbf{7 0}$ & $\mathbf{1 0 0 , 0}$ \\
\hline
\end{tabular}

Notas: SM = Salário Mínimo; *Valor do SM em 2018 = R\$ 954,00. 
Em estudo realizado por Lehmann et al. (2016) sobre a avaliação do conhecimento de gestantes e puérperas sobre a toxoplasmose na cidade do Rio Grande - RS foi identificado que $55,0 \%$ das mulheres estavam na faixa etária inferior a 25 anos.

No que se refere à cor ou raça, $60,0 \%$ das gestantes se autodeclararam como sendo pardas (Tabela 1). De igual modo, na pesquisa de Arruda et al. (2019) sobre toxoplasmose entre gestantes atendidas em uma unidade da atenção primária de Rio Branco - Acre também verificou-se maior frequência de mulheres de cor parda (73,0\%).

Em relação à escolaridade, $60,0 \%$ das mulheres tinha o ensino médio (Tabela 1). Da mesma forma, no trabalho desenvolvido por Rodrigues et al. (2015) sobre conhecimento de gestantes sobre toxoplasmose, no município de Teresina - PI, no ano de 2014 , foi encontrado que a maioria das gestantes $(70,0 \%)$ também tinham apenas o ensino médio. Com relação à situação conjugal, a maioria das mulheres declarou ter companheiro $(71,4 \%)$, conforme tabela 1 . Da mesma maneira, no estudo de Arruda et al. (2019) 64,0\% das gestantes tinham companheiro.

No que diz respeito à situação econômica, $68,6 \%$ das gestantes não exerciam a atividade remunerada e $42,9 \%$ tinham a renda familiar maior que um salário mínimo, conforme tabela 1. Este resultado é inferior ao encontrado por Lehmann et al. (2016) que identificaram que $74,7 \%$ das gestantes tinham renda familiar acima de um salário mínimo.

Na pesquisa realizada por Pavan et al. (2016) sobre as possíveis associações da positividade para a toxoplasmose com variáveis socioeconômicas, comportamentais e ambientais, identificou-se a pouca idade e grau de instrução como fatores de risco para a doença na fase gestacional.

De igual modo, no estudo desenvolvido por Moura et al. (2016) no intuito de analisar os fatores associados ao conhecimento sobre a toxoplasmose entre gestantes atendidas na rede pública de saúde do município de Niterói - RJ identificou-se a relação entre o conhecimento sobre toxoplasmose, idade e escolaridade, pois $42,7 \%$ das participantes conheciam a toxoplasmose e a proporção de gestantes com conhecimento adequado aumentou com a idade e a escolaridade, com significância estatística $(p<0,001)$.

De acordo com Barbosa (2008), o fator de risco mais importante relacionado à infecção pelo $T$. gondii são as características sociodemográficas, tendo em vista que as baixas condições socioeconômicas e escolaridade possibilitam a infecção no período gravídico devido ao menor conhecimento a respeito da doença e a existência de condições ambientais e comportamentais favoráveis.

Conforme a tabela 2, a maioria das gestantes desconhecia às formas de transmissão $(51,4 \%)$, complicações $(72,9 \%)$, meios de diagnóstico $(57,1 \%)$, profilaxia e tratamento $(74,3 \%)$ e as medidas preventivas $(52,9 \%)$ da toxoplasmose. Da mesma maneira, Millar et al. (2014) ao realizarem um levantamento do conhecimento de 400 gestantes e puérperas atendidas na rede pública de saúde do município de Niterói - RJ sobre a toxoplasmose verificaram que $72,2 \%$ das participantes nunca tinham ouvido falar sobre a toxoplasmose, principalmente sobre as formas de prevenção da infecção pelo $T$. gondii.

De forma semelhante, Rodrigues et al. (2015) e Lehmann et al. (2016) também verificaram que a maioria das gestantes entrevistadas não sabia sobre a existência e/ou risco da doença, desconheciam suas formas de infecção e medidas preventivas. Segundo Moura et al. (2018) a falta de conhecimento, a respeito da 
toxoplasmose interfere de maneira direta nas medidas de prevenção primárias. Neste sentido, Santos et al. (2018) relatam que é fundamental uma melhor educação da população, sobretudo para os grupos de maior risco, para conscientização e entendimento que uma boa higiene alimentar e pessoal são, sem dúvidas, os melhores métodos profiláticos contra a toxoplasmose. De acordo com Amendoeira e Camillo-Coura (2010) o conhecimento adequado das medidas de prevenção reduzem o risco de infecção congênita pelo $T$. gondii.

TABELA 2 - Conhecimento de gestantes sobre toxoplasmose em uma unidade de saúde de Rio Branco, Acre, Brasil, 2018.

\begin{tabular}{lcc}
\hline Variável & N & $\%$ \\
\hline Formas de transmissão & 34 & 48,6 \\
Sim & 36 & 51,4 \\
Não & & \\
Complicações da doença & 19 & 27,1 \\
Sim & 51 & 72,9 \\
Não & & \\
Meios de diagnósticos & 30 & 42,9 \\
Sim & 40 & 57,1 \\
Não & & \\
Profilaxia e tratamento & 18 & 25,7 \\
Sim & 52 & 74,3 \\
Não & & \\
Medidas preventivas & 33 & 47,1 \\
Sim & 37 & 52,9 \\
Não & & \\
Orientações sobre a toxoplasmose na gestação & 27 & 38,6 \\
Sim & 43 & 61,4 \\
Não & $\mathbf{7 0}$ & $\mathbf{1 0 0 , 0}$ \\
\hline Total & & \\
\hline
\end{tabular}

No que se refere à assistência pré-natal, $61,4 \%$ das grávidas não receberam orientações sobre a doença durante as consultas de pré-natal (Tabela 2). Também em estudo realizado por Branco et al. (2012), com o objetivo de avaliar o conhecimento e atitudes de profissionais e gestantes do serviço público de saúde sobre a toxoplasmose em unidades básicas de saúde da cidade de Maringá - PR foi identificado que apenas $16,23 \%$ das participantes relataram ter recebido informações sobre prevenção da toxoplasmose durante a gestação. Quanto aos profissionais de saúde, $39,4 \%$ não souberam responder em qual período gestacional pode-se adquirir a toxoplasmose e $15,1 \%$ não sabiam que orientações profiláticas deveriam ser dadas às gestantes.

Da mesma maneira, Cardoso et al. (2018) encontraram que a maioria das gestantes revelaram que não receberam orientações sobre a doença no seu prénatal de origem, muito preocupadas com a sua saúde e do seu bebê foram buscar informações na internet, porém algumas não entendiam as informações que buscavam.

Para Moura et al. (2018), o desconhecimento das gestantes sobre a toxoplasmose está relacionado à falta de orientações e informações dos 
profissionais de saúde durante as consultas de pré-natal, o que justifica os hábitos de vida e alimentares inadequados favorecendo uma possível infecção. Segundo Costa et al. (2017), a assistência pré-natal necessita ser reformulada não só em relação ao treinamento da equipe de assistência, que deve aprender a conhecer melhor a toxoplasmose e encontrar meio de fácil compreensão das informações pelas grávidas, assim como às unidades de saúde também devem assegurar a realização da triagem sorológica para a doença durante todos os trimestres gestacionais.

\section{CONCLUSÕES}

Os resultados deste estudo apontam que existe entre as gestantes falta de informação em relação a diversos aspectos importantes da toxoplasmose, sobretudo sobre suas formas de transmissão e medidas de prevenção. Esse cenário pode contribuir para o risco de contaminação por $T$. gondii, haja vista que as gestantes também apresentaram características sociodemográficas favoráveis à ocorrência da doença.

Dessa forma, é fundamental que os profissionais e os serviços de saúde estabeleçam ações e medidas de educação sanitária durante o pré-natal que auxiliem na orientação adequada das gestantes sobre os principais meios de transmissão e controle da toxoplasmose.

\section{REFERÊNCIAS}

AMENDOEIRA, M. R. R.; CAMILLO-COURA, L. F. Uma breve revisão sobre toxoplasmose na gestação. Scientia Medica, v.20, n.1, p.113-119, 2010. Disponível em:

<http://revistaseletronicas.pucrs.br/scientiamedica/ojs/index.php/scientiamedica/articl e/view/5917/4953>.

ARRUDA, E. F.; LIMA, A. R. M.; CARIOCA JÚNIOR, H. R.; LIRA, S. J. M.; MAIA, R. R. P. promovendo ações educativas sobre toxoplasmose entre gestantes atendidas em uma unidade da atenção primária: relato de experiência. In: SOUZA, O. F. (Org.). Saúde Pública na Amazônia Ocidental. Rio Branco: Strictu Sensu, 2019. p.67-76 Disponível em: <https://sseditora.com.br/wp-content/uploads/Saúde-Pública-naAmazônia-Ocidental.pdf>.

BARBOSA, I. R. Estudo epidemiológico da Toxoplasmose em gestantes atendidas na Maternidade Escola Januário Cicco, Natal, Rio Grande do Norte. 2008. 76 f. Dissertação (Mestrado em Biodiversidade; Biologia Estrutural e Funcional) - Universidade Federal do Rio Grande do Norte, Natal, 2008. Disponível em: <https://repositorio.ufrn.br/jspui/handle/123456789/18537>.

BRANCO, B. H. M.; ARAÚJO, S. M.; FALAVIGNA-GUILHERME, A. L. Prevenção primária da toxoplasmose: conhecimento e atitudes de profissionais de saúde e gestantes do serviço público de Maringá, estado do Paraná. Scientia Medica, v.22, n.4, p.185-190, 2012. Disponível em: <http://revistaseletronicas.pucrs.br/scientiamedica/ojs/index.php/scientiamedica/articl e/view/11718/8615>. 
BRASIL. Doenças infecciosas e parasitárias: guia de bolso. 8 ed. Brasília: Ministério da Saúde. 2010. 444 p. Disponível em: $<$ http://bvsms.saude.gov.br/bvs/publicacoes/doencas_infecciosas_parasitaria_guia_b olso.pdf>.

BRASIL. Protocolo de Notificação e Investigação: Toxoplasmose gestacional e congênita. Brasília: Ministério da Saúde, 2018. 31 p. Disponível em: $<$ http://bvsms.saude.gov.br/bvs/publicacoes/protocolo_notificacao_toxoplasmose_ge stacional.pdf>.

CARDOSO, A. C. G.; SANTOS, A. N.; GUIMARAES, J. V.; POMPEU, H. H. F. A.; COELHO, E. C. S. et al. Tecnologia educacional sobre toxoplasmose para gestantes do pré-natal de alto risco. Revista Eletrônica Acervo Saúde, v.11, n.1, p.1-10, 2018. Disponível em: <https://doi.org/10.25248/reas.e179.2019>.

COSTA, A. C.; SILVA, E. G.; ROCHA, M. G. DOMINGUES, R. J. S.; SANTOS, S. N. et al. Conhecimento sobre a toxoplasmose e associação com os fatores de risco pelas parturientes de um hospital de referência maternoinfantil. Revista Interdisciplinar de Estudos em Saúde, v.6, n.1, p.50-60, 2017. Disponível em: $<$ https://periodicos.uniarp.edu.br/ries/article/view/897>.

IBGE - Instituto Brasileiro de Geografia e Estatística. Rio Branco. 2017. Disponível em: <https://cidades.ibge.gov.br/brasil/ac/rio-branco/panorama>.

LEHMANN, L. M.; SANTOS, P. C.; SCAINI, C. J. Evaluation of pregnant and postpartum women's knowledge about toxoplasmosis in Rio Grande - RS, Brazil. Revista Brasileira de Ginecologia e Obstetrícia, v.38, p.538-544, 2016. Disponível em: <https://doi.org/10.1055/s-0036-1593970>.

MILLAR, P. R.; MOURA, F. L.; BASTOS, O. M. P.; MATTOS, D.P. B. G.; FONSECA, A. B. M. et al. Toxoplasmosis-related knowledge among pregnant and postpartum women attended in public health units in Niterói, Rio de Janeiro, Brazil. Revista do Instituto de Medicina Tropical de São Paulo, v.56, n.5, p.433-438, 2014. Disponível em: <https://doi.org/10.1590/S0036-46652014000500011>.

MONTOYA, J. G.; REMINGTON, J. S. Management of Toxoplasma gondii infection during pregnancy. Clinical Infectious Diseases, v.47, p.554-66, 2008. Disponível em: <https://doi.org/10.1086/590149>.

MOURA, F. L.; GOULART, P. R. M.; MOURA, A. P. P.; SOUZA, T. S.; FONSECA, A. $B$. M. et al. Fatores associados ao conhecimento sobre a toxoplasmose entre gestantes atendidas na rede pública de saúde do município de Niterói, Rio de Janeiro, 2013-2015. Epidemiologia e Serviços de Saúde, v.25, n.3, p.655-661, 2016. Disponível em: <https://doi.org/10.5123/S1679-49742016000300022>.

MOURA, D. S.; OLIVEIRA, R. C. M.; MATOS-ROCHA, T. J. Toxoplasmose gestacional: perfil epidemiológico e conhecimentos das gestantes atendidas na unidade básica de saúde de um município alagoano. Arquivos Médicos dos Hospitais e da Faculdade de Ciências Médicas da Santa Casa de São Paulo, 
v.63, n.2, p.69-76, 2018. Disponível em: <https: //doi.org/10.26432/18093019.2018.63.2.69>.

PAVAN, A. A.; MERLINI, L. S.; BETANIN, V.; SOUZA, E. O.; CAETANO, I. C. S. et al. Soroepidemiologia da toxoplasmose em gestantes do município de Medianeira, Paraná, Brasil. Arquivos de Ciências da Saúde da UNIPAR, v. 20, n. 2, p, 131135, 2016. Disponível em: <https://doi.org/10.25110/arqsaude.v20i2.2016.5635>.

RODRIGUES, J. B.; NASCIMENTO, L. L.; VIEIRA, P. S.; ROCHA, R. M. M.; FREITAS, D. R. J. et al. Conhecimento de gestantes sobre Toxoplasmose no município de Teresina, Piauí. Revista Prevenção de Infecção e Saúde, v.1, n.2, p.41-46, 2015. Disponível em: <https://doi.org/10.26694/repis.v1i2.3661>.

SANTOS, E. W.; SOUZA, S. L.; PEREIRA, C. A. D. Gato x gestante. Avaliação do conhecimento da população sobre a toxoplasmose. PUBVET, v.12, n.12, a238, p.15, 2018. Disponível em: <https://doi.org/10.31533/pubvet.v12n12a238.1-5>. 